\title{
Heartbeat: alcohol and the heart
}

The effects of alcohol on the heart have always been of interest for the cardiology community, with reports of both beneficial and deleterious effects. Alcoholic cardiomyopathy (ACM) is a specific form of dilated cardiomyopathy linked to chronic heavy alcohol use. In this issue of Heart, Manthey and colleagues ${ }^{1}$ used modelling strategies to estimate sexspecific mortality rates due to ACM for all countries in the world, which were subsequently aggregated by region and globally. Input data on ACM mortality were obtained from death registries for 91 countries and estimated by regression equations in the others. Overall, there were 25997 deaths worldwide due to ACM in 2015, with a mortality rate estimated at 4.9 deaths per 1000000 adults (women: 2.1; men: 7.6). The distribution of deaths was markedly uneven, with nearly $80 \%$ occurring in Central and Eastern Europe, particularly in Russia, which has the highest estimated mortality rate of 163.8 per 1000000 population (figure 1).

Under-reporting (ie, recorded deaths were lower than predicted lower limits) was identified in 44 out of 91 countries with ACM mortality data. Under-reported fatalities were concentrated in only a few countries with $43.1 \%$ of potentially under-reported deaths in Romania, Serbia and France. To some extent, the observed pattern of ACM deaths reflects the global pattern in alcohol-attributable disease burden. However, the described variability between high-burden and low-burden countries is much greater than expected compared with other alcohol-attributable causes of deaths. A possible explanation for this observation may be that ACM typically occurs with very heavy alcohol consumption over an extended period of time. The authors conclude that their data show that this lethal condition is underdiagnosed and undertreated currently, emphasising the need for routine screening and interventions to reduce the alcoholattributable mortality burden.

\footnotetext{
${ }^{1}$ Internal Medicine, Universidade Federal de Minas Gerais, Belo Horizonte, Brazil

${ }^{2}$ Division of Cardiology, University of Washington, Seattle, Washington, USA
}

Correspondence to Professor Catherine M Otto, Division of Cardiology, University of Washington, Seattle WA 98195, USA; cmotto@uw.edu
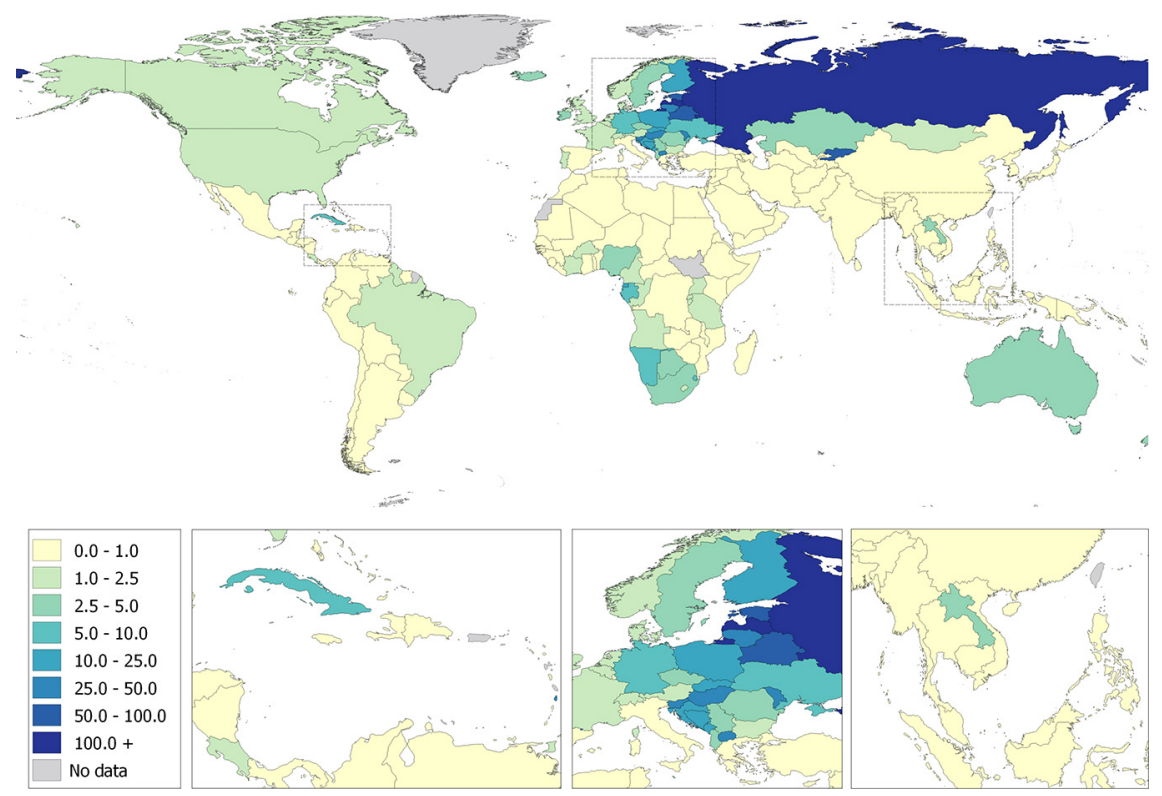

Figure 1 Alcoholic cardiomyopathy mortality rates (deaths per 1000000 adult population) in 2015. Mortality rates denote the number of deaths per 1000000 adult population.

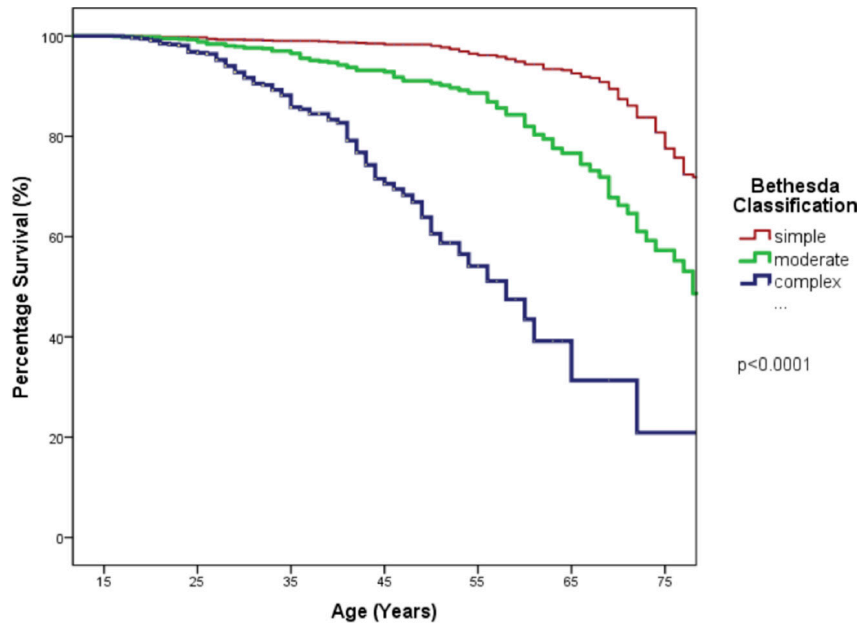

\begin{tabular}{|l|l|l|l|l|l|l|l|}
\hline CHD type & $\mathbf{1 6 y}$ & $\mathbf{2 5 y}$ & $\mathbf{3 5 y}$ & $\mathbf{4 5 y}$ & $\mathbf{5 5 y}$ & $\mathbf{6 5 y}$ & $\mathbf{7 5 y}$ \\
\hline Simple & 1452 & 1374 & 1103 & 848 & 641 & 420 & 199 \\
\hline Moderate & 1029 & 905 & 554 & 281 & 158 & 76 & 29 \\
\hline Complex & 545 & 452 & 226 & 72 & 20 & 5 & 1 \\
\hline
\end{tabular}

Figure 2 Kaplan-Meier survival curves arranged by severity of underlying adult congenital heart disease (ACHD).

In their editorial, Rohde and Beck-daSilva $^{2}$ discuss 'the good, the bad and the worse' related to ACM. Specifically, they point out that advocating a cardioprotective role for alcohol may be risky, considering all the dangers related to alcoholic beverages and heart disease.
Life expectancy in adults with congenital heart disease (ACHD) has improved dramatically over the past few decades. In a cohort of over 3000 Australian patients with CHD with a median follow-up of 6.2 years, the incidence of death was 0.4 per 100 patient-years. $^{3}$ As expected, mortality 


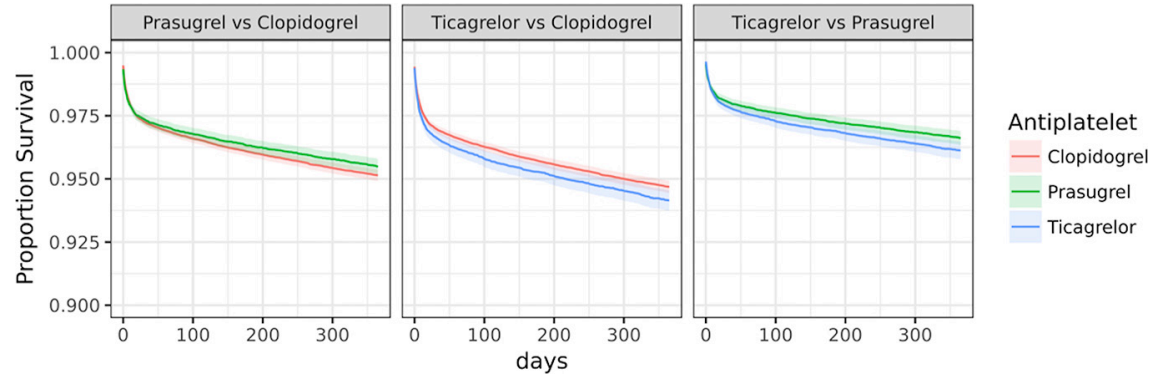

Figure 3 Kaplan-Meier curves for the propensity-score-matched datasets. Cls are represented by coloured shades.

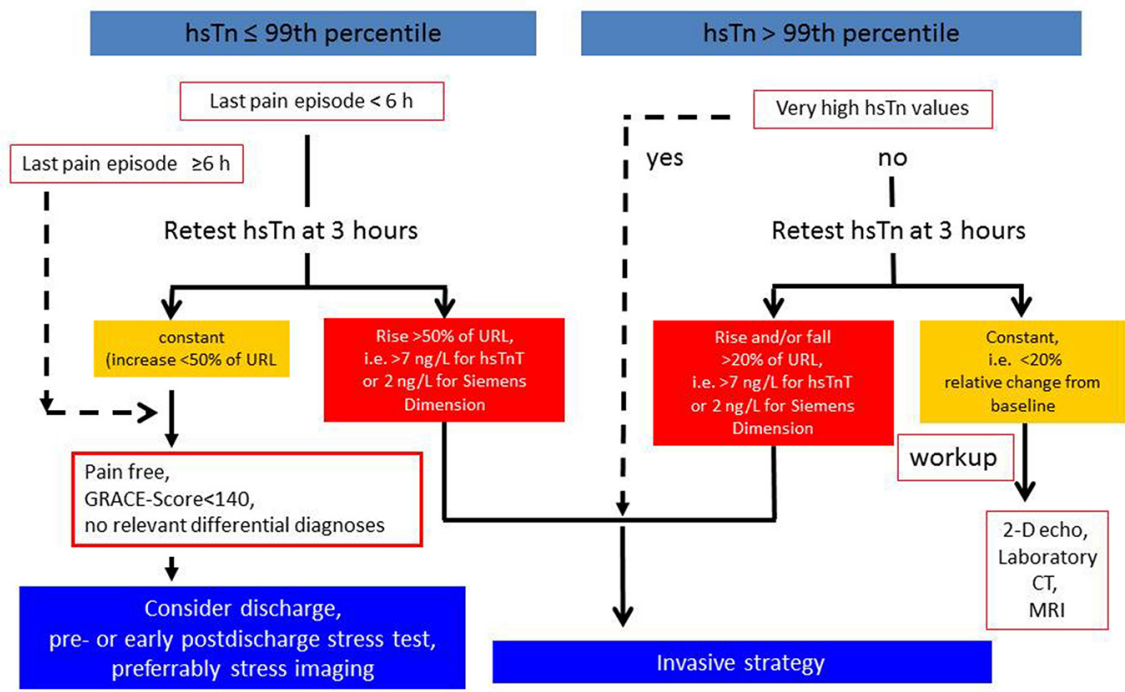

Figure 4 The 2015 ESC guideline recommended a 0/3-hour diagnostic algorithm if the highsensitivity troponin (hsTn) assay is available. URL, upper reference limit. ESC, European Society of Cardiology; GRACE, Global Registry of Acute Coronary Events.

was higher withincreasing complexity of ACHD, ranging from 0.3 deaths $/ 100$ patient-years with a median age at death of 70 years in the $47 \%$ with simple ACHD to as high as 1.0 deaths/100 patient-years with median age at death of 34 years in the $18 \%$ of patients with complex CHD (figure 2). Interestingly, although heart failure was the single most common cause of death ( $17 \%$ of deaths), overall non-cardiac causes accounted for $54 \%$ of deaths.

In an accompanying editorial, Buber and Valente $^{4}$ point out the challenges in understanding ACHD survival data including the heterogenous diagnoses included in this broad category, the lack of evidence-based standardised medical therapy for these conditions, the variety of surgical and transcatheter procedures for correction or palliation of the congenital lesions, and the difficulty in accurately determining the cause of death in many cases. They argue that we need better standardisation of data for defining outcomes in patients with ACHD. 'Redefining the manner in which we consider levels of risk in ACHD patients focused on more than their birth congenital diagnosis and considers additional factors, such as genomics, biomarkers and functional status will most likely result in better planning of healthcare system utilisation and targeted innovations in interventional and medical therapies for the populations who remain at the greatest risk of early mortality and morbidity.'

In another interesting study in this issue, Olier and colleagues ${ }^{5}$ used data from over 89000 patients in the British Cardiovascular Interventional Society national database to compare outcomes in patients treated with ticagrelor, prasugrel or clopidogrel after primary percutaneous coronary intervention for acute ST-elevation myocardial infarction (STEMI). Their main multivariate logistic regression analysis showed a significantly lower mortality with prasugrel compared with clopidogrel at 30 days and 1 year (OR 0.89, 95\% CI 0.82 to $0.97, p=0.011)$. There was no difference in mortality when ticagrelor was compared with clopidogrel and a higher mortality with ticagrelor compared with prasugrel (figure 3).

In an editorial commenting on this study, Nijjer' ${ }^{6}$ concludes that 'The data by Olier et al should give pause to those still using clopidogrel as their default drug in STEMI. The move from prasugrel to ticagrelor is less clear-cut and the data presented here are provocative.' However, 'This question is not fully solved by the present data set, which can only generate hypotheses. Future studies are awaited, such as the ISAR-REACT 5 study ('Prospective, Randomized Trial of Ticagrelor Versus Prasugrel in Patients With Acute Coronary Syndrome') which will randomise 4000 patients in Europe to ticagrelor or prasugrel but it will include all ACS (acute coronary syndrome) rather than STEMI alone, and is still recruiting.

The Education in Heart article in this issue $^{7}$ provides a quick overview of the use of troponin levels in diagnosis of myocardial infraction, as well as in other clinical settings (figure 4). Try the Image Challenge ${ }^{8}$ to see if you can make the diagnosis, based on CT imaging and ECG findings, in a bike rider who hit a tree.

Competing interests None declared.

Patient consent Not required.

Provenance and peer review Commissioned; internally peer reviewed.

(c) Author(s) (or their employer(s)) 2018. No commercial re-use. See rights and permissions. Published by BMJ.

\section{Check for updates}

To cite Ribeiro TL, Otto CM. Heart

2018:104:1639-1640.

Heart 2018;104:1639-1640.

doi:10.1136/heartjnl-2018-314119

\section{REFERENCES}

1 Manthey J, Probst C, Rylett M, et al. National, regional and global mortality due to alcoholic cardiomyopathy in 2015. Heart 2018;104:1663-9.

2 Rohde LE, Beck-da-Silva L. Alcohol and the heart: the good, the bad and the worse in heart failure. Heart 2018;104:1641-2

3 Yu C, Moore BM, Kotchetkova I, et al. Causes of death in a contemporary adult congenital heart disease cohort. Heart 2018;104:1678-82.

4 Buber J, Valente AM. Predicting survival in adults with congenital heart disease: what are the odds? Heart 2018; 104:1643-4.

5 Olier I, Sirker A, Hildick-Smith DJR, et al. Association of different antiplatelet therapies with mortality after primary percutaneous coronary intervention. Heart 2018; 104:1683-90.

6 Nijjer SS. Clinical outcomes of potent antiplatelets compared with clopidogrel in ST elevation myocardial infarction. Heart 2018:104:1645-6.

7 Giannitsis E, Katus HA. Troponins: established and novel indications in the management of cardiovascular disease. Heart 2018;104:1714-22.

8 Spapen J, Lochy S, Michiels V. A shocking bike accident. Heart 2018;104:1706. 